\title{
Introducing the special focus issue: cellular reprogramming and iPS cells
}

\section{"Is iPS cell technology the future of clinical medicine? Read on, you will certainly not be disappointed!"}

We would like to welcome you to this special focus issue of Regenerative Medicine on cellular reprogramming and induced pluripotent stem (iPS) cells. The likely impact of iPS cells on the field of regenerative medicine has been much debated, and our aim has been to separate hype from hope in order to give a true picture of the potential and limitations of this exciting scientific breakthrough.

It is no exaggeration to state that iPS cells are a highly disruptive technology, and certainly as a direct consequence for clinical medicine "the future ain't what it used to be" [1]. Therefore, the greatest challenge for the Editorial Team and Guest Editors was to not only present a robust foundation to the topic, but equally importantly, its destiny.

Selecting what is relevant needs a clear vision of the future. Alan Kay, the eminent US computer scientist once remarked that, "The best way to predict the future is to invent it. Really smart people with reasonable funding can do just about anything that doesn't violate too many of Newton's Laws!" [101]. Therefore, who better to preside over the contents of this iPS cell special focus issue than two exceptional leaders who are both internationally recognized to be at the very forefront of the field, Willy Lensch (Harvard University, MA, USA) and Mahendra Rao (Invitrogen, CA, USA). Their perception, insight and genuine enthusiasm for this advanced cell-based technology is unquestionable.

In particular, we felt it was important that our Guest Editors, and the issue as a whole, represented both academic and industry perspectives and, most importantly, their integration. As the Guest Editors themselves point out, the translation of this technology will require the fostering of "the cooperative interplay between basic research institutions, biotech and pharmaceutical companies" [2]; a view that we the Regenerative Medicine Editorial Team wholeheartedly endorse.

The two Guest Editors have expertly identified the relevant key topics and brought onboard authors that represent the pinnacle of the growing mountain of talented stakeholders who, with adequate funding, are ideally positioned to unlock the medical potential of iPS cell by transforming this technology into genuine benefits for patients. The result is a special issue that is sharply focussed, up to date and contains outstanding content with real impact - a true 101 guide to the fast-moving world of iPS cell technology.

In closing, we would like once again to thank our Guest Editors, Mahendra Rao and Willy Lensch, as well as all of the authors and reviewers who have contributed to the issue. We trust that this iPS cell special focus issue will both educate and entertain, plus through its breadth of diverse expert perspectives, give a clear overall vision of the future for this formidable leadingedge technology. Is iPS cell technology the future of clinical medicine? Read on, you will certainly not be disappointed!

\section{References}

1 Yogi Berra (1925-) - American baseball player. The Oxford Dictionary of Modern Quotations (2nd Edition). Knowles E (Ed.). Oxford University Press, UK (2002).

2 Lensch MW, Rao M: Induced pluripotent stem cell: opportunities and challenges. Regen. Med. 5(4), 483-484 (2010).

\section{- Website}

101 Alan Kay (1940-): "The best way to predict the future is to invent it" www.smalltalk.org/alankay.html

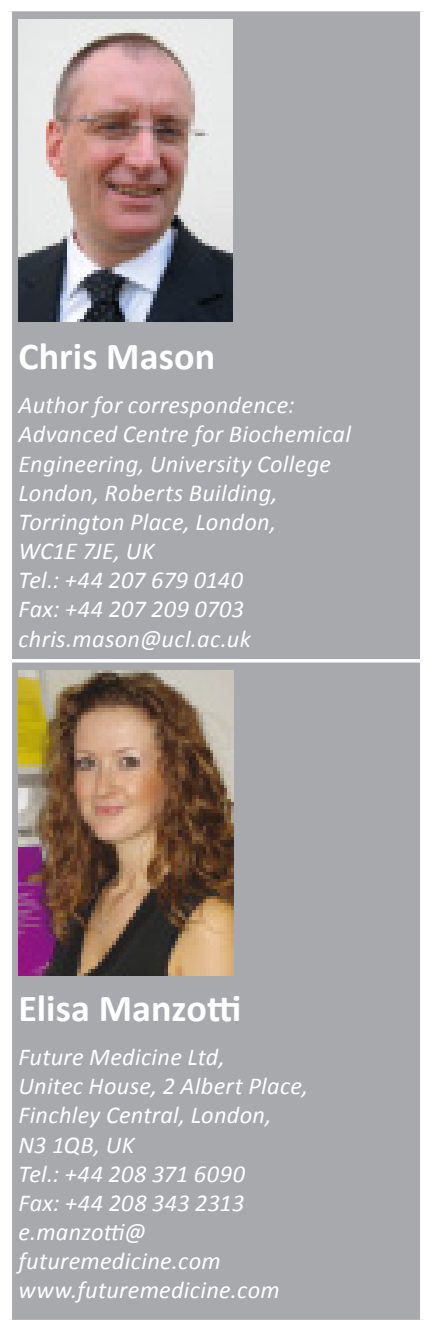

\section{future medicine $^{\text {part }}$ fs}

